\title{
Modelling Of PAFC Based Scattering Monitoring System for the Characterization of the Therapeutic Micro-Bubbles
}

\author{
Vibhor K. Bhardwaj* and Surita Maini \\ Department of Electrical and Instrumentation Engineering, Sant Longowal Institute of Engineering \& Technology, \\ Punjab 148106, India
}

* e-mail: vibhorkrbhardwaj@gmail.com

\begin{abstract}
In recent years, researchers are eagerly developing the Ultrasound Cavity Agents (UCA) as a therapeutic agent, so that they can deliver the drugs to an intended place in a guided manner with minimal invasiveness and maximum effectiveness. However, control dissolution of the drug is still an issue because the shell of the micro-bubble sometimes collapses instantaneously and start releasing the drug at a faster rate. This sudden rise in the pressure's level can rupture the capillaries and sometimes blood vessels also. Therefore, in such cases, it is a great challenge to examine the dynamics of the micro-bubble as well as the health of the blood vessel. In this essence, this paper presents a study based on the finite element method to examine the potential use of Photo-Acoustic Flow Cyclometery (PAFC) to resolve this issue. The presented model is based on the study of the intensity variations of the optical scatterings engender by the microbubbles through PAFC. The results of the study reveal that the proposed model has the potential to be used as a new mechanism to examine the growth of the micro-bubble as well as the health of the blood vessel. Moreover, by analysing the scattering pattern, one can also able to predict the value of the cavitation threshold and the size of the micro-bubble. Hence, the authors envisioned that the modified PAFC system can lead the path of a low-cost and real-time examiner for accurate target drug delivery as well as for the health of the blood vessel. Which in turn potentially increases the localized concentration of the drug to reduce the concerned side-effects of medicine on the other part of the body. (C) 2019 Journal of Biomedical Photonics \& Engineering.
\end{abstract}

Keywords: therapeutic agents; microbubble; PAFC; FEM; cavitation threshold.

Paper \#3335 received 6 Aug 2019; revised manuscript received 26 Sep 2019; accepted for publication 26 Sep 2019; published online 30 Sep 2019. doi: 10.18287/JBPE19.05.030303.

\section{Introduction}

In the present decade, the advances of biomedical science have focused the research in the new minimalinvasive method for targeted drug delivery. Compare to conventional therapies this method provides a strict localization of the drugs at the vascular pathology sites. Therefore, a significant improvement has been observed in therapeutic efficacy without any drug toxicity and systematic side-effects. In recent years, researchers are eagerly discovering the potential use of ultrasound contrast agents (UCAs) as a transporter to deliver the drug at a specific location. Due to their small size (mean diameter of 1.1 to $3.3 \mu \mathrm{m}$ ), they can easily travel into small peripheral capillaries of blood along with bloodstream [1-3]. In contrast to its primary role as a diagnostic tool to improve imaging modality of the small vessels of the human organs, they have potential to be used as a therapeutic agent having drugs in their core [4]. These therapeutic agents have potential application in the treatment of a certain type of cancer, thrombolysis [5-7], real-time and non-invasive blood pressure measurement [8] during surgery and many more.

Till now various studies have been conducted to understand the interaction of bubble with a blood vessel, 
determination of the size of the bubbles, the evolution of the bubble size, effect of resonance on the bubble, and discrimination of bubbles with other particles, etc. [9-12]. But still, there are many challenges, which must be addressed before making it a tested and trusted tool. Moreover, their further therapeutic capabilities are still being explored by several research groups in different countries, but the results are not yet concluded.

These challenges, however, propelling the researchers towards a new field of bubble dynamics to understand the therapeutic capability of the microbubble for different ailments in the different organs. Furthermore, the behaviour and characterization of the bubbles are also an important aspect for the medical imaging, therapy, and diagnostic field.

Commonly, the characterization of the microbubble is conducted by the mean of measuring the acoustic scattering and attenuations. However, this method is unable to characterize the free micro-bubbles due to the limitation of acoustic resolution and the bandwidth of the acoustic receivers $[13,14]$. Therefore, for the characterization of an individual microbubble, optical imaging techniques have been used consisting of highspeed cameras and streak cameras. But, this technique produces a huge amount of processed data, thus limiting its operation only for few-cycle of ultrasound $[15,16]$.

To overcome this problem, another alternative approach for characterization is based on the analysis of scattered light $[17,18]$. This method not only reduces the amount of data per oscillation cycle but also capable to characterize the dynamics of the whole microbubble population. As compare to high-speed imaging, this method is unable to provide direct visual information about the dynamics of the microbubble. But, low cost and ease of incorporation with ultrasound devices make this method more promising for further research [19].

Considering the above challenges, the authors present a Photo Acoustic Flow Cytometer (PAFC) based model for real-time observation of the microbubble dynamics. In this model, the PAFC has been used to create acoustic pressure that expands the microbubble shell and thereby providing an intensity variation in the scattered light. Thus, by analysing the scattering pattern, one can also be able to predict the value of the cavitation threshold and the size of the micro-bubble. The study has also presented mathematical modelling along with a finite element modelling of the proposed model. The results of the study reveal that the proposed model has the potential to provide a label-free rapidassessment of the micro-bubble dynamics to manage adequate intravascular pressure in real time. Moreover, the health of the blood vessel can also be assessed in real time by comparing the reference signal. However, the clinical trials are a more accurate analysis of the system but for examining the feasibility of a novel approach, the computational models have substantial advantages for understanding and translating the outcome.

The system is modelled in COMSOL Multiphysics in the two-dimensional mode. Thus, a realistic finite element representation of the practical problem can be done using coupled physics modelling.

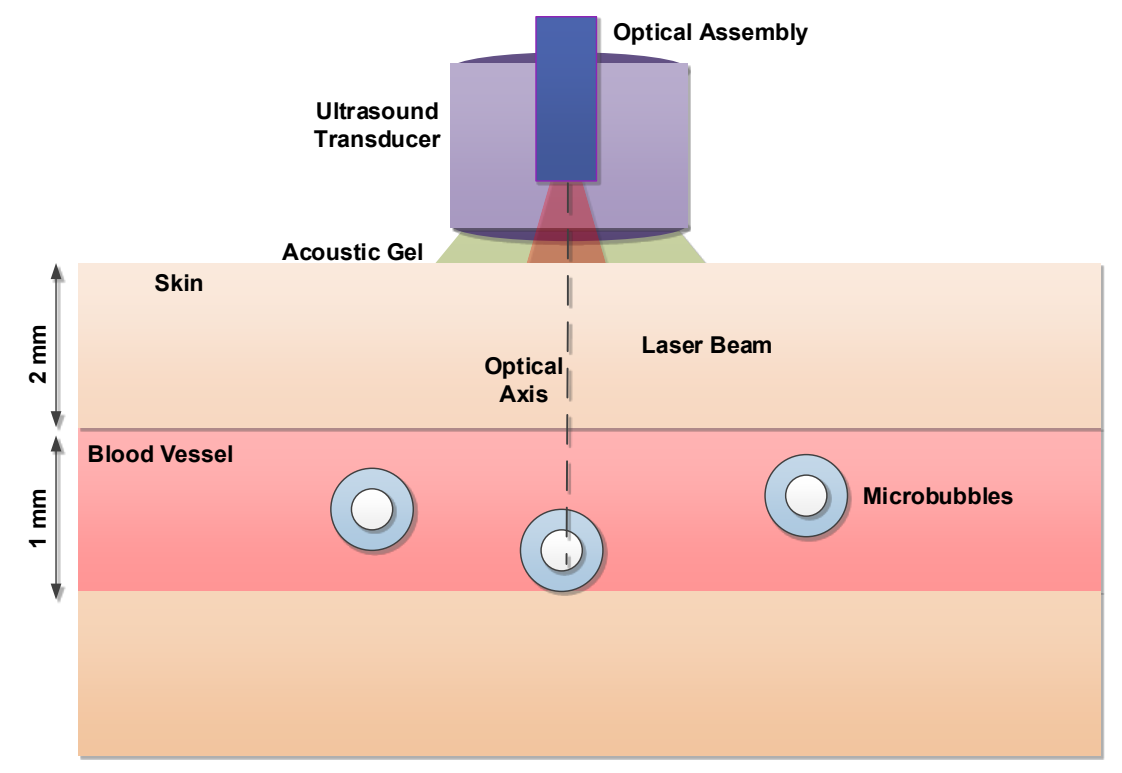

Fig. 1 Illustration of PAFC setup consist of a $4 \mathrm{~mm}$ disc-shape ultrasound transducer and a $1024 \mathrm{~nm}$ laser beam (fed through a $0.2 \mathrm{~mm}$ core multi-mode optical fibre). The numerical aperture (NA) of the transducer assembly is 0.5 to capture wide-angle PA excitation, while the arterial wall has a thickness of $0.2 \mathrm{~mm}$.

\section{Method and model description}

Conventional flow cytometry (FC) is being used as a popular biological tool for many years [20]. This type of tools is based on the principle of scattering and fluorescent of the laser beam. A more advanced version of FC is a combination of laser and ultrasound techniques and known as photo-acoustic (PA) flow 
cytometry (PAFC). This photo-acoustic device enhances the quality of the signal for both the optical and acoustic mechanism. It enhances the laser's sensitivity along with spectral specificity and side by side also improves spatial resolution and depth of penetration for ultrasound [21]. Therefore, by using PAFC a more accurate observation of micro-bubbles size and evolution can be performed, and a more feasible method can be made to manage adequate intravascular pressure for bursting. An illustration of the proposed model is shown in Fig. 1. The ultrasound transducer is used to create acoustic pressure on the micro-bubbles and the laser, and its supporting components are used to examine the real-time behaviour of the micro-bubble. A $1 \mathrm{MHz}$ ultrasound transducer was modelled to detect the laser-induced acoustic waves based on the work of Andrews [22]. While the laser diode was modelled to emit the pulses having $10 \mathrm{~ns}$ pulse-width with $10 \mathrm{kHz}$ of reception rate at $1064 \mathrm{~nm}$ wavelength. The detail discussion on working principle of PAFC setup is available in several literatures [23, 24]. To ensure the proper bonding between the skin and the ultrasound transducer, typically acoustic gel is used as a conductive medium. However, this acoustic gel absorbed a portion of the emitted laser beam with an absorption coefficient of $0.65 \mathrm{~cm}^{-1}$ [25].

\subsection{Light scattering and absorption}

When light is targeted on a surface, some portion of the light gets extinction due to absorption and scattering. In the case of micro-bubble, this extinction depends on the size of the micro-bubble, thickness of the shell and refractive index of core/shell of the microbubble [26]. Various theories have been developed to explain the scattering of light from the microspheres [27-29]. For example, typical mathematical model for the diffraction of light can be developed using Maxwell's equations in spherical coordinates [30]. However, for ease of understanding, a simple mathematical model is formulated with the assumption that extinction of light in the medium is due to the absorption and scattering only:

$$
\sigma_{e x t}=\sigma_{a b s}+\sigma_{s c a}
$$

where $\sigma_{\text {ext }}, \sigma_{a b s}, \sigma_{s c a}$ are the coefficients of extinction, absorption and scattering, respectively. Furthermore, using Mie theory of scattering, if a wave of " $\lambda$ " wavelength having " $I_{o}$ " intensity is transmitted through a medium and after transmission " $P$ " intensity wave is received then absorption " $A$ " by the medium can be defined as:

$$
A=-\log _{10}\left(\frac{I}{I_{o}}\right)
$$

Here, it is also an interesting point that the intensity of scattered light also depends on the angle of observation, extinction coefficient, path length "l" and number density " $N$ " [30-32]:

$$
I=I_{o} e^{-\sigma_{e x t} l N}
$$

Thus, contribution in light extinction by the absorption can be expressed as:

$$
A=\frac{l N}{2.3} \sigma_{\text {ext }}
$$

The absorption in the homogenous medium having a concentration of " $c$ " and molar extinction coefficient " $\varepsilon$ " can also be defined using Beer-Lambert law as [33]:

$$
A=\varepsilon c l \text {. }
$$

Therefore, from Eqs. (4) and (5), the extinction coefficient can be expressed as:

$$
\sigma_{e x t}=2.3 \frac{\varepsilon c}{N}
$$

\subsection{Bubble Discrimination}

Globally, there are various commercially available UCA agents which have the potential to be used as a carrier for target drug delivery, like Definity, Optison, Imagify, Sonazoid, Targestar, and MicroMarker. The properties of UCA agents such as shell coating, core-gas, and mean diameters are core parameters, which are required to be studied before implementing in real time situations are listed in Table 1.

Table 1 List of Commercially available ultrasound contrast agents with their manufacture name, coating material and gas filled inside the core.

\begin{tabular}{r|rrrr}
\multicolumn{1}{r}{ UCA } & Manufacture by & Shell Coating & Core Gas & $\begin{array}{r}\text { Mean diameter } \\
(\mu \mathrm{m})\end{array}$ \\
\hline Definity & Lantheus Medical & Phospho-lipids & C3F8 & $1.1-3.3$ \\
Optison & Imaging & Human albumin & C3F8 & $3.0-4.5$ \\
Imagify* & GE Healthcare & Polymer & C4F10 & $1.9-2.2$ \\
Sonazoid & Acusphere & Daiichi Pharmaceutical & C4F10 & $2.5-3.0$ \\
Targestar & Targeson & Phospho-lipids & C4F10 & $3.0-4.5$ \\
MicroMarker & Bracco & Fatty Acids & C4F10 & $2.3-2.9$
\end{tabular}


The core of these UCAs, are generally filled with perfluoropropane (C3F8) or perfluorobutane (C4F10) gas and the outer shell is coated to stabilize them so that they can survive long enough in the body without prebursting. It is worth to note that long survival time is also a great challenge for microbubbles. Because they are highly compressed compare to blood and tissue layers and their compressibility makes them more scattering element, which is a boon to recognize them. The maximum diameter of these agents has been recorded up to $32 \mu \mathrm{m}$ but generally, the mean diameter varies between 1.1 to $4.5 \mu \mathrm{m}$. However, the selection of the UCA depends on the type of targeted organ and its associated capillary $(5 \mu \mathrm{m})$, artery $\left(10^{3} \mu \mathrm{m}\right)$ and arteriole $(20 \mu \mathrm{m})$ of the human body.

In this paper, Definity has been taken as a reference micro-bubble because of its small and consistently-size with extensive safety experience, which also makes it a prominently used therapeutic agent. Moreover, it is worth to note here that these microbubbles can also be used to measure the amount of blood delivered in a tissue per unit time. In literature, the sub-harmonic oscillations of the microbubble are described by the Church-Hoff equation [34]. This equation describes the behavior of the micro-bubble with the following assumption:

1. There is a zero-surface tension between the microbubbles shell and the blood interface.

2. The overall distribution of the microbubbles shell thickness is constant.

3. There is no effect of the driving frequency and acoustic pressure on the behavior of the viscoelastic material used for the micro-bubble formation.

With such assumptions, the linear resonance frequency $\left(f_{o}\right)$ of the micro-bubble for the ultrasound region can be described by:

$$
f_{o}=\frac{\omega_{o}}{2 \pi}=\frac{1}{2 \pi R_{e}} \sqrt{\frac{1}{\rho_{B}}}\left(3 k P_{o}+12 G_{s} \frac{d_{s}}{R_{e}}\right) .
$$

Moreover, for the incompressible flow of blood, the acoustic pressure $\left(P_{S}\right)$ scattered by the microbubble can be given as [35]:

$$
P_{s}(t)=\rho_{B}\left(2 v_{w}^{2}+R a_{w}\right)
$$

where $R_{e}$ is the equilibrium radius of the micro-bubble, $\rho_{B}$ is the density of blood, $k$ is the poly-tropic exponent, $P_{o}$ is the atrial blood pressure, $G_{s}$ is the shear modulus of micro-bubble shell, $d_{s}$ is the thickness of the microbubble shell, $v_{w}$ is the wall velocity of microbubble, $R$ is the instantaneous radius of the microbubble and $a_{w}$ is the acceleration of micro-bubble wall.

From the above two Eqs. (7) and (8), the conclusion can be drawn that the instantaneous radius of the microbubble under the influence of the ultrasound signal is one of the influencing parameters for both resonance frequency and acoustic pressure used for the bursting.

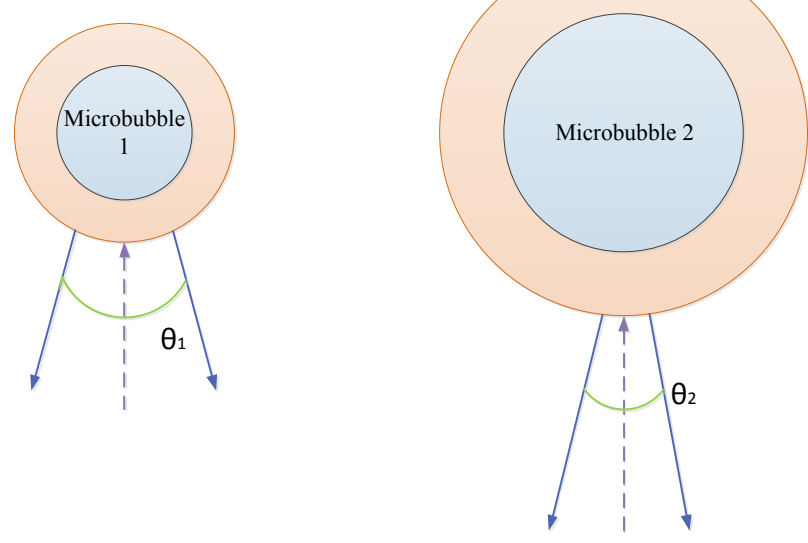

Fig. 2 Indicating the angular distribution of reflected power by two different size microbubbles. The change in the total reflected power occurs due to induction of the different divergence angle $(\theta)$. The beam axis has been denoted by a broken line and reflection by a solid line.

Furthermore, the spherical shape of the microbubbles produce a partially diffuse reflection (assumed as $0.1 \mathrm{~cm}^{-1}$ [36]) thereby scattering intensity follows the exponential relationship with the position of the beam axis [37]. Moreover, as the micro-bubble grows, the curvature of the surface decreases. This subsequently concentrate the laser intensity over a small angle of the divergence angle $(\theta)$. Thus, light reflected from a small bubble have less intensity compare to a bubble having higher diameter as illustrated in Fig. 2. Therefore, power distribution of light over the angular range can be used to detect the size or growth of the micro-bubble. Chen et al. [38] modelled the scattering behaviour of the encapsulated microbubble in 2009. The same has been referred in the paper.

\section{Boundary Descriptions for Finite Element Analysis}

\subsection{Boundary Conditions for Microbubble}

The model is defined with the following boundary conditions at different interfaces to satisfy the churchHoff equation is shown in Fig. 3. Furthermore, for a better understanding of dynamics, Marmottant model [39] has been used to characterize the micro-bubble.

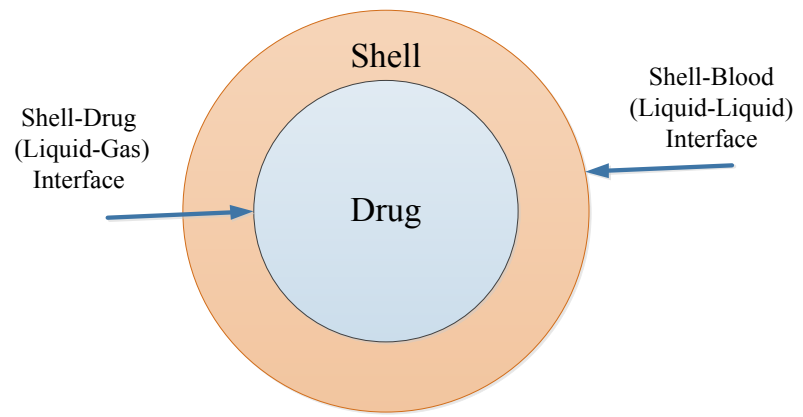

Fig. 3 Illustration of different interfaces of microbubble. 
a) Shell-Drug Interface:

Usually, the drug-filled in the micro-bubble is in form of gas [40] and the stabilizing shell is made up of liquids like lipids or proteins and fluorinated gases with a high molecular weight [41]. For this reason, the boundary conditions used for this interface should be followed both the kinematic and dynamic nature of the interface. Therefore, the dynamics conditions have been used to describe the zero-shear stress at the interface while the kinematic condition ensures the stability of the microbubble. Moreover, if the volume of the drug $\left(V_{g}\right)$ inside the micro-bubble remains constant and the gas (drug) satisfying the poly-tropic relation as explained in the equation (7) to determine the linear resonance frequency, then we can define, the relationship between the partial pressure inside the micro-bubble $\left(P_{g}\right)$ and the volume of the $\left(V_{g}\right)$ as

$$
P_{g} V_{g}^{k}=\text { constant }
$$

\section{b) Shell-Blood Interface:}

This interface is defined by dynamic boundary conditions because both the shell and blood are in the liquid state. These dynamic conditions are responsible for providing continuity for both shear stresses and movement of blood and microbubble [42]. Furthermore, interface surface tension, density, perfusion rate, temperature, velocity, pressure, and viscosities of both the medium are also defined by this boundary condition. The parameters used for the modelling are listed in Table 2.

Table 2 Modelling parameter defined for the blood and the artery.

\section{Comput.} Domain

\begin{tabular}{l|ll}
\hline \multirow{5}{*}{ Artery } & Initial Value Modulus & $1.74[\mathrm{MPa}]$ \\
& Initial Bulk Modulus & $34.7[\mathrm{MPa}]$ \\
& Young Modulus & $6.2[\mathrm{MPa}]$ \\
& Density & $103\left[\mathrm{~kg} / \mathrm{m}^{3}\right]$ \\
& Poisson's Ratio & 0.45 \\
& Model Material & Hyperelastic \\
& & Neo-Hookean \\
& & \\
& Density & $1060\left[\mathrm{~kg} / \mathrm{m}^{3}\right]$ \\
& Perfusion Rate & $3.6 \times 10^{-3}[1 / \mathrm{sec}]$ \\
& Temperature & $37\left[{ }^{\circ} \mathrm{C}\right]$ \\
& Viscosity & $3.5[\mathrm{MPa}-\mathrm{sec}]$ \\
& Velocity & $1 \times 10-2[\mathrm{~m} / \mathrm{sec}]$ \\
& Pressure & $12[\mathrm{kPa}]$ \\
& Model Material & Incompressible \\
& & Navier-Stokes
\end{tabular}

\section{c) Blood-Skin Interface:}

For modelling the blood, a steady flow velocity profile is defined at the inner side of the boundary. The artery is assumed to be non-slip and believed that the blood will not penetrate the wall. Therefore, the velocity of blood flow is uniform and considered to be laminar. The artery parameter like initial shear and bulk modulus, Young modulus, density, Poisson's ratio used for modelling is also listed in Table 2. The absorption coefficient $\left(\sigma_{a b s}\right)$ and scattering coefficient $\left(\sigma_{s c a}\right)$ of skin used for the modeling are 0.37 and $1 \mathrm{~cm}^{-1}$ respectively [43].

\subsection{Boundary Conditions for the Rest of the Model}

The boundary conditions used for the modeling are depicted in Fig. 4. Because only a section of the human blood vessel has been modelled therefore it becomes necessary to maintain the symmetry of the computational domain to the rest of the domain. So, to make boundaries identical on both the sides of the computational domain Floquet periodic boundary conditions has been applied on both left and right sides of the model. To launch the laser beam, periodic port boundary conditions has been used rather than diffraction order port conditions. Because due roughness of the surfaces, if diffraction order port is used to monitor the reflected and transmitted light, hundreds (or thousands) of diffraction orders are received, which only increases computational complexity. This model is designed only for statistical sampling, hence the relative fraction of laser beam scattered by the microbubble by these different orders has not been considered. Therefore, to compute the reflection and transmission, a perfectly matched layer (PML) is defined above and below of the model and placed at least half a wavelength away to avoid absorption of evanescent field component. Therefore, by using the PMLs we can compute all the unimpeded reflections and avoid using multiple ports to compute total transmittance. Lastly, to evaluate total transmitted and reflected light two additional interior boundaries are placed near the PMLs. These boundaries have been used to integrate the power flux created in both upward and downward directions and are normalized it by the incident power. It is here worth to note that, the acoustic pressure beyond the tolerance of the micro-bubble shell can destruct it instantaneously, and the release of gas with such pressure that can damage adjacent area like blood vessels/capillaries. Thus, it becomes mandatory to apply controlled pressure on the bubble and a systematic incremental in the pressure should be applied with the observation in the bubble growth. The microbubbles considered in this study of the size $1-2,4-5,6-8,8-10$ and $10-12 \mu \mathrm{m}$ are driven at a frequency of $1 \mathrm{MHz}$ with a pressure range of $95 \mathrm{kPa}$ to $333 \mathrm{kPa}$. In any case, if the pressure increases beyond $333 \mathrm{kPa}$ before target bursting, it may lead to severe complications. The prior bursting reasons may be the different diameter of the bubble, circumstances of the vessel/capillary of the subject, physiochemical properties of blood, different blood pressures, any blockage in a blood vessel and other complications. 


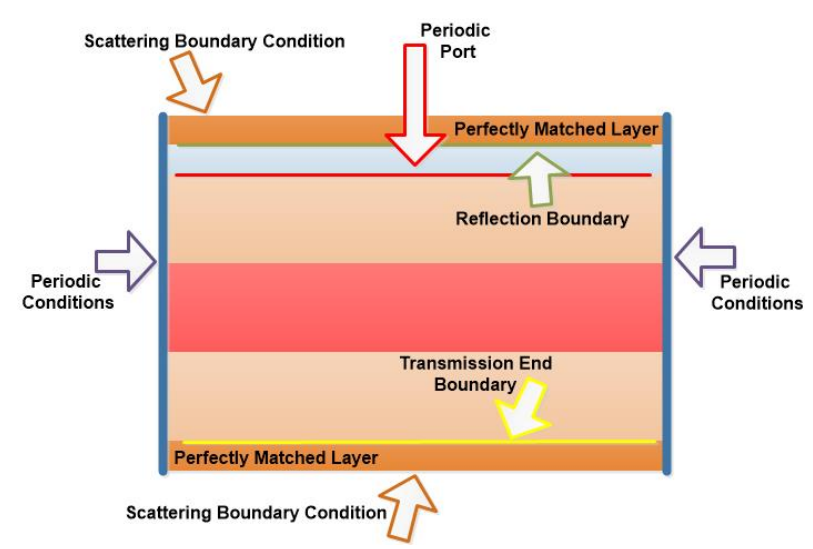

Fig. 4 Boundary conditions for the rest of the model.

\section{Results and Discussions}

The relative intensity of the light scattered by the microbubble obtained by the proposed model is depicted in Fig. 5 in which an articulate expansion and disruption in the micro-bubble diameter engender by the acoustic pressure are shown. A continuous fluctuating signal is also noticed during the whole time-period (250 $\mathrm{sec}$ ) of measurement that may be considered as the optical signature of the blood or other properties. During this time-period, a $1 \mathrm{MHz}$ acoustic signal with 15 cycles per pulse has been induced after every $30 \mathrm{sec}$. Thus, a total of 9 shots of ultrasound pulses are targeted. For each pulse, when a shot of photo-acoustic signal is transmitted with a higher intensity of the laser beam, data has been collected and plotted. Thus, the observation of the bubble growth is observed after every $30 \mathrm{sec}$. This pulsating measurement technique also helps to avoid unwanted side-effects caused by a higher intensity of light and enable us to be imaging little deep. Acoustic pressure up to $333 \mathrm{kPa}$ is non-destructive in nature for the microbubble, and growth of the microbubble is observed as an increment in the peak amplitude. However, if this pressure level is increased further, the inner shell (the core) of the bubble is collapsed, and leakage of gas started. This leakage in gas make the bubble dormant towards the acoustical pressure and thus further expansion in the bubble is ceased. This event is marked with the increment in the base fluctuating signal after 4 pulse. Therefore, a controlled diffusion in the gas become necessary to prevent the blood vessel to get ruptured. Therefore, corresponding to the cavitation threshold, pressure should be imposed on the micro-bubble.

To determine the cavitation threshold by the scattering of light, a micro-bubble having mean diameter has been considered as a reference bubble to characterize the growth of other individual bubbles. Thus, a non-linearity in the intensity of the scattered light is observed as compared to the reference scattering with the drift in the acoustic pressure towards the cavitation threshold. This non-linearity is caused, due to the asymmetric expansion in the bubble engender by the bursting of the inner shell of the bubble. Moreover, in context to the reference scattering of the light intensity, the size of the bubble can be estimated. Working in the direction of discrimination of the micro-bubbles. Therefore, authors classified the bubbles in two segments based on the reference diameter. Fig. 6 illustrated a scenario that represents the discrimination among the micro-bubble having different diameter compared to the reference diameter i.e. $5 \mu \mathrm{m}$ in this case. One can easily distinguish the individual microbubble by observing the gap size presented between the spikes. This individual discrimination is based on the absorption of the light beam rather than the scattering of light. Therefore, whenever the bubble crosses the optical axis the peaks get extinction.

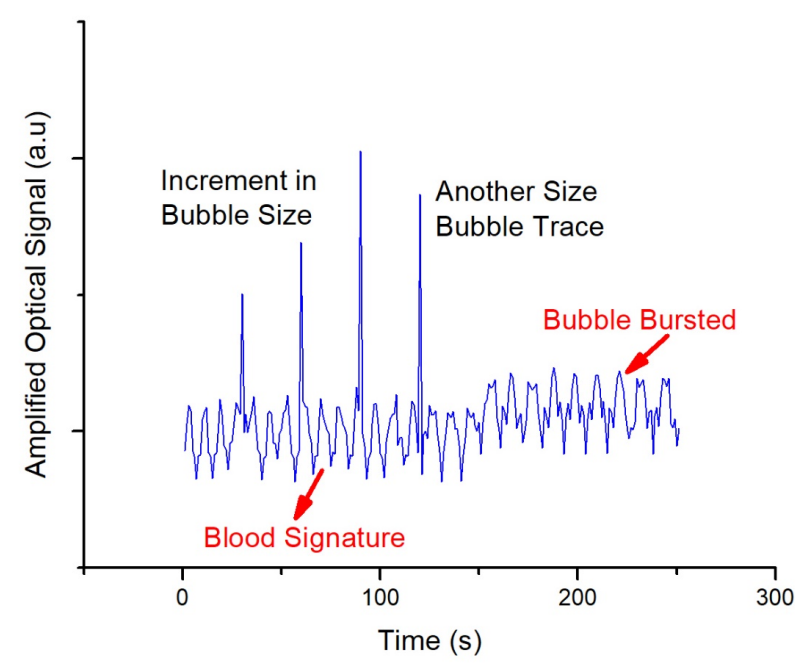

Fig. 5 Plot indicating the growth of the bubble after every shot of ultrasound signal. Fluctuating signals are also been reported in the plot, which may be considered as the signature of blood. Furthermore, bursting of microbubble is also recorded as the increment in the signal level.

This classification is necessary to understand the variation in the scattering pattern, as it is well known that the scattering is based upon the suspended particles. Thus, as the size of the microbubbles is increased, a unique scattering pattern will be observed in Fig. 6. This scattering pattern may further used to estimate the stretching in the outer lipid shell of the bubble, which in turn help to calculate the pressure level at the surface of the individual bubbles. Here, it is worth to note that the latency between the ultrasound pulses and the optical scattering creates ambiguity in the microbubble characterizations. So, to avoid this post-signalprocessing issue it becomes necessary to achieve a high Signal to Noise Ratio (SNR). Hence, a high-intensity laser should be used with low noise detectors. Thus, it is envisioned that the PAFC type modelling for the characterization of microbubble dynamics have higher potential compared to other scattering based tools.

But still, there is a challenge to perform scanning in the large blood vessels because there is a trade-off between the beam width and the background noise. It may be possible that some bubbles, which are circulating in the blood vessel may get missed by the 
laser beam. Therefore, this method is only suitable for the superficial blood vessels having small diameter so that a preferable optical environment (low tissue absorption) can be obtained. Furthermore, in case of deep sealed vessels, there is a high tissue absorption rate, hence scattered light intensity is attenuated largely, and optical resolution is reduced. Thus, it is concluded that, for superficial blood vessel, this method has the potential to characterize the micro-bubble dynamics, which allow monitoring the pressure level and cavitation threshold.

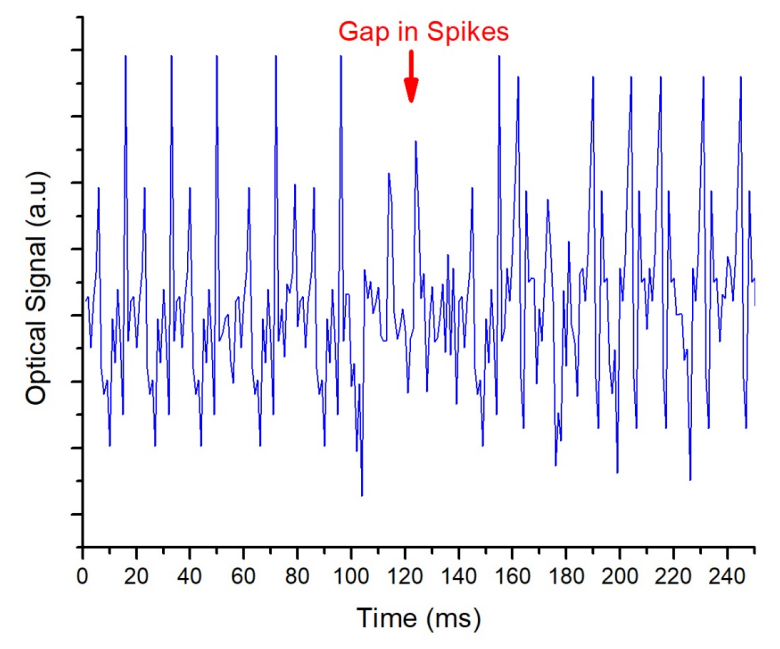

(a)

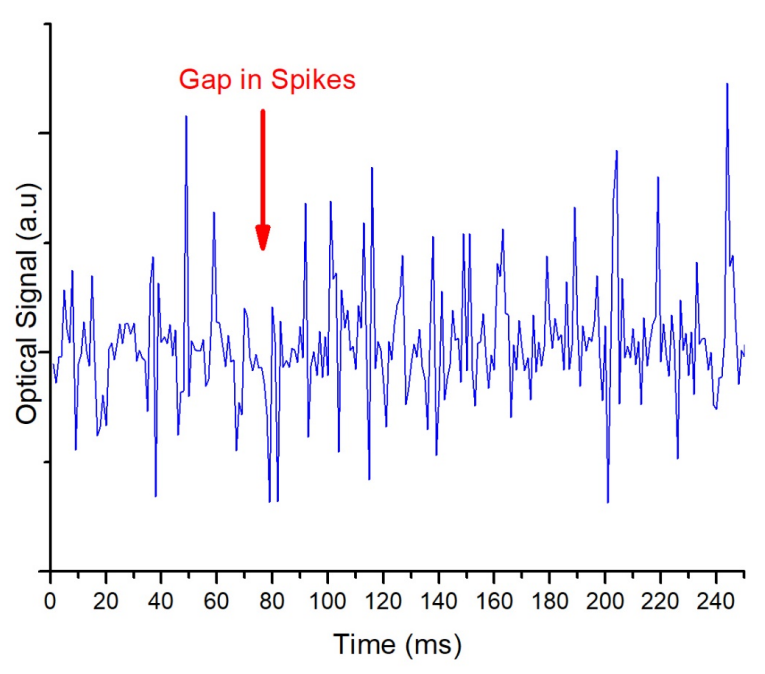

(b)

Fig. 6 Plots indicating the presence of microbubble having size (a) greater than $5 \mu \mathrm{m}$; (b) less than $5 \mu \mathrm{m}$. The size of the microbubble present at the foot print of the optical beam has been estimated by analysing the gap in the spikes.

Moreover, as we all are aware that only $10 \%$ of the antibiotics are consumed by the human body and rest $90 \%$ are excreted into the environment thereby affecting the ecosystem of the earth and sea creatures. Thus, by using such technique the optimum volume of drugs can be targeted towards the affected organ, that will not only reduce the recovery period of ailment but also minimize the side-effect on the other organs as well as the ecosystem. All the scientists and the researchers working on this field are contributing their baby-steps towards this new technique of drug delivery. However, epslon changes by each researcher will create a big impact in this direction. Finally, the authors have tried to contribute an epslon contribution in this side of research, although it is a drop-in ocean, authors hope that it will beneficial for all the researchers contributing in this direction.

\section{Conclusion and Future Scope}

A framework to characterize the dynamics of the microbubbles through analysing the intensity of the optical scattering by the PAFC system has been presented in this study. A multi-physics finite element method-based model is designed to examine the growth of the microbubbles under the influence of the acoustic pressure up to $333 \mathrm{kPa}$ with $1 \mathrm{MHz}$ frequency. The proposed model has indicated better efficacy in the case of the superficial blood vessel. Although, in the case of deep sealed blood vessels, authors have observed a reduction in the optical resolutions due to increment in the optical scattering losses. Another limitation of PAFC model is that the system only accounted for the microbubble that came into the vicinity of the laser. Although, most of the micro-bubbles, which are freely circulating in the vessel, remains out of the vicinity of the optical axis and did not contribute any optical scattering. In contrast, they have only produced most of the acoustic echoes and affecting the SNR of the model. However, authors have tried to improve the SNR by increasing the laser intensity level but faced the trade-off between different parameters. Therefore, this limitation of efficacy for deep sealed blood vessels and freely circulating microbubbles needs to be addressed in the future by increasing the SNR of the system or any other suitable technique. Despite above-mentioned constraints, the authors envisioned that the proposed model will provide low-cost, effective and less-complex estimation for characterization of the therapeutic micro-bubbles in case of superficial blood vessels. Furthermore, this technique unveils the secret of drug delivery that can help to reduce the side effects of medicine overdose.

\section{Disclosures}

All authors declare that there is no conflict of interests in this paper. 


\section{References}

1. C. A. Sennoga, E. Kanbar, L.Auboire, P.-A. Dujardin, D. Fouan, J.-M. Escoffre, and A. Bouakaz, "Microbubblemediated ultrasound drug-delivery and therapeutic monitoring," Expert Opinion on Drug Delivery 14(9), 10311043 (2017).

2. J. M Tsutsui, F. Xie, and R. T. Porter, "The use of microbubbles to target drug delivery," Cardiovascular Ultrasound 2(1), 23 (2004).

3. G. Shapiro, A. W. Wong, M. Bez, F. Yang, S. Tam, L. Even, D. Sheyn, S. Ben-David, W. Tawackoli, G. Pelled, K. W. Ferrara, and D. Gazit, "Multiparameter evaluation of in vivo gene delivery using ultrasound-guided, microbubble-enhanced sonoporation," Journal of Controlled Release 223, 157-164 (2016).

4. A. A. Doinikov, A. Bouakaz, "Modeling of the dynamics of microbubble contrast agents in ultrasonic medicine: Survey," Journal of Applied Mechanics and Technical Physics 54(6), 867-876 (2013).

5. J. E. Leeman, J. S. Kim, F. T. H. Yu, X. Chen, K. Kim, J. Wang, X. Chen, F. S. Villanueva, and J. J. Pacella, "Effect of Acoustic Conditions on Microbubble-Mediated Microvascular Sonothrombolysis," Ultrasound in Medicine \& Biology 38(9), 1589-1598 (2012).

6. S. Datta, C.-C. Coussios, A. Y. Ammi, T. D. Mast, G. M. de Courten-Myers, and C. K. Holland, "UltrasoundEnhanced Thrombolysis Using Definity ${ }^{\circledR}$ as a Cavitation Nucleation Agent," Ultrasound in Medicine \& Biology 34(9), 1424-1433 (2008).

7. S. M. Chowdhury, T. Lee, and J. K. Willmann, "Ultrasound-guided drug delivery in cancer," Ultrasonography 36(3), 171-184 (2017).

8. F. Li, L. Wang, Y. Fan, and D. Li, "Simulation of noninvasive blood pressure estimation using ultrasound contrast agent microbubbles", IEEE Transactions on Ultrasonics, Ferroelectrics and Frequency Control 59(4), 715-726 (2012).

9. M. Postema, H. Abraham, O. Krejcar, and D. Assefa, "Size determination of microbubbles in optical microscopy: a best-case scenario," Optics Express 25(26), 33588 (2017).

10. S. Li, Y. Qin, X. Wang, and X. Yang, "Bubble growth in cylindrically-shaped optical absorbers during photomediated ultrasound therapy," Physics in Medicine \& Biology 63(12), 125017.

11. C. Harfield, C. R. Fury, G. Memoli, P. Jones, N. Ovenden, and E. Stride, "Analysis of the Uncertainty in Microbubble Characterization," Ultrasound in Medicine \& Biology 42(6), 1412-1418 (2016).

12. K. Efthymiou, N. Pelekasis, M. B. Butler, D. H. Thomas, and V. Sboros, "The effect of resonance on transient microbubble acoustic response: Experimental observations and numerical simulations," The Journal of the Acoustical Society of America 143(3), 1392-1406 (2018).

13. J. Sijl, B. Dollet, M. Overvelde, V. Garbin, T. Rozendal, N. de Jong, D. Lohse, and M. Versluis, "Subharmonic behavior of phospholipid-coated ultrasound contrast agent microbubbles," The Journal of the Acoustical Society of America 128(5), 3239-3252 (2010).

14. S. Paul, A. Katiyar, K. Sarkar, D. Chatterjee, W. T. Shi, and F. Forsberg, "Material characterization of the encapsulation of an ultrasound contrast microbubble and its subharmonic response: Strain-softening interfacial elasticity model," The Journal of the Acoustical Society of America 127(6), 3864-3857 (2010).

15. V. Garbin, D. Cojoc, E. Ferrari, E. Di Fabrizio, M. L. J. Overvelde, S. M. van der Meer, N. de Jong, D. Lohse, and M. Versluis, "Changes in microbubble dynamics near a boundary revealed by combined optical micromanipulation and high-speed imaging," Applied Physics Letters 90(11), 114103 (2007).

16. J. E. Chomas, P. A. Dayton, D. May, J. Allen, A. Klibanov, and K. Ferrara, "Optical observation of contrast agent destruction," Applied Physics Letters 77(7), 1056 (2000).

17. F. Urgiles, J. Perchoux, and T. Bosch, "Characterization of Acoustic Sources by Optical Feedback Interferometry," Proceedings 1(4), 348 (2017).

18. M. J. Hsu, M. Eghtedari, A. P. Goodwin, D. J. Hall, R. F. Mattrey, and S. C. Esener, "Characterization of individual ultrasound microbubble dynamics with a light-scattering system," Journal of Biomedical Optics 16(6), $067002(2011)$.

19. J. F. Guan, T. J. Matula, "Using light scattering to measure the response of individual ultrasound contrast microbubbles subjected to pulsed ultrasound in vitro," The Journal of the Acoustical Society of America 116(5), 2832-2842 (2004).

20. E. I. Galanzha, V. P. Zharov, "Photoacoustic flow cytometry," Methods 55(3), 280-296, (2012).

21. M. A. Juratli, Y. A. Menyaev, M. Sarimollaoglu, A. V. Melerzanov, D. A. Nedosekin, W. C. Culp, J. Y. Suen, E. I. Galanzha, and V. P. Zharov, "Noninvasive label-free detection of circulating white and red blood clots in deep vessels with a focused photoacoustic probe,” Biomedical Optics Express 9(11), 5667-5677 (2018).

22. D. Andrews, "Modelling of Ultrasonic Transducers and Ultrasonic Wave Propagation for Commercial Applications using Finite Elements with Experimental Visualization of Waves for Validation," Proceedings of the 2014 COMSOL Conference in Cambridge (2014). 
23. E. I. Galanzha, M. G. Viegas, T. I. Malinsky, A. V. Melerzanov, M. A. Juratli, M. Sarimollaoglu, D. A. Nedosekin, and V. P. Zharov, "In vivo acoustic and photoacoustic focusing of circulating cells," Scientific Reports 6(1), 1-15 (2016).

24. M. A. Juratli, Y. A. Menyaev, M. Sarimollaoglu, E. R. Siegel, D. A. Nedosekin, J. Y. Suen, A. V. Melerzanov, T. A. Juratli, E. I. Galanzha, and V. P. Zharov, "Real-Time Label-Free Embolus Detection Using In Vivo Photoacoustic Flow Cytometry," PLOS One 11(5), e0156269 (2016).

25. L. C. Cabrelli, P. I. B. G. B. Pelissari, A. M. Deana, A. A. O. Carneiro, and T. Z. Pavan, "Stable phantom materials for ultrasound and optical imaging," Physics in Medicine and Biology 62(2), 432-447 (2017).

26. M. Kerker, The scattering of light and other electromagnetic radiation, Academic press, New York (1969).

27. F. M. Kahnert, "Numerical methods in electromagnetic scattering theory," Journal of Quantitative Spectroscopy and Radiative Transfer 79-80, 775-824 (2003).

28. T. Wriedt, “A Review of Elastic Light Scattering Theories," Particle \& Particle Systems Characterization 15(2), 67-74 (1998).

29. V. Myroshnychenko, J. Rodríguez-Fernández, I. Pastoriza-Santos, A. M. Funston, C. Novo, P. Mulvaney, L. M. Liz-Marzán, and F. J. G. de Abajo, "Modelling the optical response of gold nanoparticles," Chemical Society Reviews 37(9), 1792-1805 (2008).

30. M. R. Rashidian Vaziri, A. Omidvar, B. Jaleh, and N. Partovi Shabestari, "Investigating the extrinsic size effect of palladium and gold spherical nanoparticles," Optical Materials 64, 413-420 (2017).

31. D. L. Kingsbury, P. L. Marston, "Mie scattering near the critical angle of bubbles in water," Journal of the Optical Society of America 71(3), 358 (1981).

32. M. A. Blizard, "Scattering Of Light By A Coated Bubble In Water Near The Critical And Brewster Scattering Angles," Proceeding of SPIE 925, 308 (1988).

33. L. Shi, L. A. Sordillo, A. Rodríguez-Contreras, and R. Alfano, "Transmission in near-infrared optical windows for deep brain imaging," Journal of Biophotonics 9(1-2), 38-43 (2016).

34. L. Hoff, P. C. Sontum, and J. M. Hovem, "Oscillations of polymeric microbubbles: Effect of the encapsulating shell," The Journal of the Acoustical Society of America 107(4), 2272-2280 (2000).

35. D. Chatterjee, K. Sarkar, "A Newtonian rheological model for the interface of microbubble contrast agents," Ultrasound in Medicine \& Biology 29(12), 1749-1757 (2003).

36. H. Assadi, R. Karshafian, and A. Douplik, "Optical scattering properties of intralipid phantom in presence of encapsulated microbubbles," International Journal of Photoenergy 2014, 1-9 (2014).

37. J. Herbert, K. Bertling, T. Taimre, A. D. Rakić, and S. Wilson, "Microparticle discrimination using laser feedback interferometry," Optics Express 26(20), 25778 (2018).

38. J. Chen, K. S. Hunter, and R. Shandas, "Wave scattering from encapsulated microbubbles subject to highfrequency ultrasound: Contribution of higher-order scattering modes," The Journal of the Acoustical Society of America 126(4), 1766 (2009).

39. P. Marmottant, S. van der Meer, M. Emmer, M. Versluis, N. de Jong, and S. Hilgenfeldt, D. Lohse, “A model for large amplitude oscillations of coated bubbles accounting for buckling and rupture," The Journal of the Acoustical Society of America 118(6), 3499-3505 (2005).

40. G. L. Chahine, C.-T. Hsiao, "Modeling Microbubble Dynamics In Biomedical Applications," Journal of Hydrodynamics 24(2), 169-183 (2012).

41. J. M. Hyvelin, E. Gaud, M. Costa, A. Helbert, P. Bussat, T. Bettinger, and P. Frinking, "Characteristics and Echogenicity of Clinical Ultrasound Contrast Agents: An in Vitro and in Vivo Comparison Study," Journal of Ultrasound in Medicine 36(5), 941-953 (2017).

42. G. L. Chahine, "Interaction Between an Oscillating Bubble and a Free Surface," Journal of Fluids Engineering 99(4), 709-716 (1977).

43. A. N. Bashkatov, E. A. Genina, V. I. Kochubey, and V. V. Tuchin, "Optical properties of human skin, subcutaneous and mucous tissues in the wavelength range from 400 to $2000 \mathrm{~nm}$," Journal of Physics D: Applied Physics 38(15), 2543-2555 (2005). 